Service social

\title{
Le savoir se construit ou quand la relève participe à la recherche en violence
}

\section{Geneviève Martin}

Volume 44, numéro 2, 1995

Visages de la violence

URI : https://id.erudit.org/iderudit/706699ar

DOI : https://doi.org/10.7202/706699ar

Aller au sommaire du numéro

Éditeur(s)

École de service social de l'Université Laval

ISSN

1708-1734 (numérique)

Découvrir la revue

Citer cet article

Martin, G. (1995). Le savoir se construit ou quand la relève participe à la recherche en violence. Service social, 44(2), 181-186.

https://doi.org/10.7202/706699ar d'utilisation que vous pouvez consulter en ligne.

https://apropos.erudit.org/fr/usagers/politique-dutilisation/ 


\section{COMMENTARES ET DOCUMENTS}

\section{Le savoir se construit - ou quand la relève participe à la recherche en violence}

Geneviève MARTIN

Professeure École de service social, Université Laval

Au moment de la parution du numéro «Par delà les barrières des sexes » en 1988, nous avons introduit cette rubrique et préparé une nomenclature de travaux féministes. Devant l'utilité reconnue de cet écrit, nous refaisons l'exercice, en recensant, cette fois, les ouvrages de $2^{\mathrm{e}}$ et de $3^{\mathrm{e}}$ cycle en service social, de 1990 à 1995, qui se rapportent à la violence familiale et à la violence faite aux femmes. Ce numéro spécial sur la violence nous paraît le lieu privilégié pour faire part de contributions originales pouvant être sources $d^{\prime}$ inspiration pour de futures recherches et expériences d'intervention.

Les documents mentionnés ici se trouvent dans les centres de documentation de l'École de service social de I'Université de Montréal et de l'École de service social de l'Université Laval. Nous avons 
regroupé les titres par thèmes et sous-thèmes pour en faciliter le repérage. En complément, nous ajoutons la liste des publications du CRI-VIFF.

\section{VIOLENCE ENVERS LES ENFANTS}

\section{Enfants négligés}

Grenier, Sylvie

Intervention en matière de protection de la jeunesse dans la problématique de la négligence au Centre des services sociaux de Québec.

Québec : Université Laval, École de service social. Essai, 1992.

NAULT, Lise

Le concept de soi des enfants négligés.

Québec: Université Laval, École de service social. Mémoire, 1991.

\section{Témoins ou victimes de violence}

CIMON, Louise

Perception du vécu des enfants témoins ou victimes de violence conjugale chez les intervenants psychosociaux et les interventions réalisées auprès des enfants et de leur famille.

Québec: Université Laval, École de service social. Mémoire, 1995.

VAILLANT, Louise L'intervention auprès des enfants témoins et/ou victimes de violence dans la famille.

Montréal : Université de Montréal, École de service social. Rapport de stage, 1992.

SECOURS, Jean-Luc Les enfants victimes de violence physique ou psychologique.

Montréal : Université de Montréal, École de service social. Rapport de stage, 1990.

\section{Enfants agressés sexuellement}

BERGERON, Réjean Le témoignage en cour criminelle de mineures victimes d'agression sexuelle. Observations des réactions des enfants victimes d'agression sexuelle durant leurs témoignages devant la chambre criminelle.

Québec: Université Laval, École de service social. Essai, 1992.

MAILloux, Sonia L'utilisation de l'activité dans le traitement de groupe d'enfants victimes d'agression sexuelle.

Québec: Université Laval, École de service social. Essai, 1991.

\section{Mères d'enfants violentés}

LANDRY, Yolande

Intervention de groupe auprès de mères d'enfants victimes d'abus sexuel intra-familial.

Québec: Université Laval, École de service social. Essai, 1992. 


\section{ADOLESCENTS}

\section{Agresseurs sexuels}

LAFOREST, Suzette Perception, par les adolescents auteurs de délits sexuels, de leur participation à une intervention de groupe.

Québec: Université Laval, École de service social. Essai, 1991.

\section{Violents}

BRODEUR, Normand Analyse de la mise sur pied d'un groupe pour adolescents violents.

Montréal : Université de Montréal, École de service social.

Rapport de stage, 1993.

HÉBERT, Jacques La prévention de l'agression juvénile : un projet-pilote. Montréal: Université de Montréal, École de service social.

Thèse, 1991.

\section{Approche interculturelle}

GOBEIL, André Les représentations sociales de la violence chez des adolescents et des adolescentes d'origines ethniques différentes de Montréal.

Québec: Université Laval, École de service social. Mémoire, 1993.

LAMARCHE, Danielle Approche interculturelle auprès de jeunes d'origine haitienne victimes de violence familiale.

Montréal : Université de Montréal, École

de service social, XIV. 137, 37 pages, 1991.

\section{ADULTES}

\section{Agresseurs sexuels}

GUÉRIN, Gervais L'évaluation de l'agresseur sexuel. Expérimentation d'un guide d'évaluation de l'abuseur sexuel auprès d'individus ayant abusé sexuellement de leurs enfants.

Québec: Université Laval, École de service social. Essai, 1991.

AlEXANDRE, Herman Analyse d'une intervention de groupe auprès de pères incestueux.

Québec: Université Laval, École de service social. Essai, 1990.

\section{Survivantes d'inceste}

DAMANT, Dominique Effets de deux programmes d'intervention pour des femmes adultes agressées sexuellement pendant l'enfance.

Québec: Université Laval, École de service social. Thèse, 1995.

NADEAU, Éliette Survivantes adultes d'inceste. Espoir et renaissance. Analyse $d^{\prime} u n$ processus de groupe.

Québec: Université Laval, École de service social. Essai, 1991. 


\section{Violence conjugale}

LEFEBVRE, Francine Violence conjugale et interventions féministes.

Montréal : Université de Montréal, École de service social.

Travail dirigé, 1993.

GRIMARD, Francine La composition d'un groupe. Réflexion critique d'une expérimentation, auprès d'un groupe de femmes victimes de violence conjugale du Centre féminin du Saguenay Inc., d'une approche de groupe en service social axée sur le développement personnel et l'entraide.

Québec: Université Laval, École de service social. Essai, 1992.

LEBCEUF, Louise Intervention en approche structurelle auprès d'Inter'elles, un réseau d'entraide pour femmes violentées et auprès $d^{\prime}$ individus et familles usagers du CSSMM.

Montréal : Université de Montréal, École de service social. Rapport de stage, 1992.

OUELLET, Hélène Femmes victimes de violence conjugale: intervention féministe en maison d'hébergement.

Montréal : Université de Montréal, École de service social.

Rapport de stage, 1992.

SIMONEAU-YERGEAU, Analyse d'une expérience d'intervention de groupe auprès Jacqueline

des femmes victimes de violence conjugale en milieu rural, axée sur l'aide mutuelle et la perspective féministe.

Québec: Université Laval, École de service social. Essai, 1990.

Tessier-Couturier, G. Analyse d'une intervention auprès d'un groupe de femmes violentées de la Côte-Nord.

Québec: Université Laval, École de service social. Essai, 1990.

\section{Violence et médiation}

AlAIN, Yvette

Pertinence de la médiation dans les cas de violence conjugale.

Montréal : Université de Montréal, École de service social.

Rapport de stage, 1993.

\section{Violence et formation}

BROWN, Brita

La mise sur pied d'un colloque de formation pour les intervenantes des maisons d'hébergement.

Montréal : Université de Montréal, École de service social.

Rapport de stage, 1993.

\section{Conjoints violents}

LefebVRe, Jean-Pierre Projet d'étude des caractéristiques des conjoints violents et des programmes d'aide leur étant destinés.

Montréal : Université de Montréal, École

de service social. Mémoire, 1990. 
DOSTIE, Pierre L'impact de la supervision sur la mobilisation des forces du groupe en réadaptation, implantation et évaluation d'un modèle de supervision de groupe au Groupe d'aide aux personnes impulsives de Québec (GAPI).

Québec: Université Laval, École de service social. Essai, 1993.

\section{VIOLENCE CONTRE LES PERSONNES ÂGÉES}

THÉRIAULT, Cécile Inventaire des perceptions de personnes âgées de 70 ans et plus fréquentant un centre de jour, sur la violence exercée envers les personnes âgées (territoire du CLSC du Centre-de-la-Mauricie).

Québec: Université Laval, École de service social. Mémoire, 1995.

\section{VIOLENCE EN DIVERS MILIEUX}

CARON, Estelle

Une perspective écologique de la violence et de la motivation en milieu scolaire.

Montréal: Université de Montréal, École de service social.

Rapport de stage, 1992.

BOURDAGES, Isabelle Stratégies d'intervention dans la relation praticien-client en contexte de violence dans un milieu psychiatrique: des pistes pour l'action et propositions d'un protocole d'intervention.

Québec: Université Laval, École de service social. Essai, 1995.

SANSCHAGRIN, Évaluation des effets d'un programme scolaire de groupe Suzanne en prévention des abus physiques et sexuels des enfants. Québec: Université Laval, Ecole de service social. Essai, 1990.

\section{ANALYSE DE PRATIQUE}

DANKWORTH, Jürgenh Consellor's Accounts of Wife Abuse and Their Clinical Practice (Explorenga Cintroversy).

Montréal : Université de Montréal, École de service social. Thèse, 1993.

RACINE, Sonia

L'évolution du concept et des pratiques de lutte telle que perçue par des militants de Centres d'aide et de lutte contre les agressions à caractère sexuel (CALACS) du Québec; 1980-1990.

Québec: Université Laval, École de service social. Mémoire, 1995. 


\section{RÉFLEXIONS}

$\mathrm{Si}$, en première analyse, on peut dégager quelques tendances de la recherche étudiante telles que, d'une part, l'intérêt pour l'intervention, pour les enfants témoins ou victimes, pour la violence conjugale, pour les aspects interculturels, en revanche le peu de regard sur la violence envers les aînés et le peu d'analyse de la pratique font qu'il nous paraît impossible de tracer adéquatement le portrait en ce domaine. Trop de travaux étudiants sont en cours dans les équipes JEFET (Jeunes et Familles en transition) et CRI-VIFF. Un bilan, auquel pourront contribuer d'autres instances universitaires et d'autres équipes, s'imposera donc sous peu, car la moisson s'annonce abondante et fort pertinente pour la compréhension de la problématique de la violence.

\section{LISTE DES PUBLICATIONS DU CRI-VIFF}

Préoccupation en émergence dans la pratique et la recherche en violence.

Collection Réflexions, $\mathrm{n}^{\circ} 1$.

Violence envers les femmes: la controverse des chiffres.

Collection Réflexions, $\mathrm{n}^{\circ} 2$.

Liens entre la violence physique, psychologique et sexuelle faite aux enfants et aux femmes.

Collection Réflexions, $\mathrm{n}^{\circ} 3$.

Utilisation des ressources par les victimes de violence conjugale.

Le cas des femmes référées aux CLSC par les policiers.

Collection Études et analyses, $\mathrm{n}^{\circ} 1$.

Exploration des principaux dilemmes éthiques associés à l'intervention auprès des conjoints violents et des stratégies pour les résoudre.

Collection Études et analyses, $n^{\circ} 2$.

L'intervention de groupe auprès des conjoints violents :

Quand l'évaluation s'allie à la pratique.

Collection Outils, $n^{\circ} 1$. 